Lep. Rev. (1968) 39, 2, 84-86

\title{
Effect of BCG Vaccination on Leprosy in Kenya
}

\author{
D. L. LEIKER \\ Royal Tropical Institute, Amsterdam, Netherlands \\ (Temporary Member of the Medical Research Centre, Nairobi, Kenya) \\ Y. OTSYULA \\ East African Leprosy Research Centre, Alupe, Kenya \\ M. ZIEDSES DES PLANTES \\ Government Leprosarium, Alupe, Kenya
}

In 1962 Dr. C. M. Ross had started a pilot leprosy control project in Samia district and he intended to study the effect of BCG vaccination on leprosy. The work of this devoted and conscientious leprologist was broken off by his death in 1964. The data of his surveys mentioned in this article are derived from his original notes.

In 1962 Ross examined 177 children in schools at Buholo, Central Nyanza. He found 28 children with leprosy, a prevalence of $16 \%$. All patients were non-lepromatous.

In 1964 the schoolchildren in this area were vaccinated with BCG. In 1966 we have carried out a re-survey in the same schools. In 320 children examined only one tuberculoid patient was found, a prevalence of $0.3 \%$.

In 1962 Ross also examined 60 children in schools at Sio Port. He found 8 non-lepromatous patients, a prevalence of $13.3 \%$. In 1964 BCG was given to the children. In 1966 we re-surveyed the schools. In 276 children examined only one tuberculoid patient was found, a prevalence of $0.4 \%$. This patient was one of the few children who were absent when the schoolchildren were vaccinated. He developed leprosy in 1965.

Of the 37 patients registered by Dr. Ross in 1962, 15 children were re-examined in 1966 . In these children the lesions had completely resolved, in many of them without treatment. Obviously the patients seen by Dr. Ross, and classified as indeterminate or tuberculoid, had a very mild self-healing type of leprosy.

\section{DISCUSSION}

It is unlikely that in the 4 years between the surveys major changes in the leprosy and in the tuberculosis situation have occurred. The decline in incidence of leprosy in the schoolchildren is probably to the greatest extent due to the BCG vaccination. The findings correspond with those of a recent BCG trial in Uganda (Kinnear Brown et al., 1966). BCG vaccination confers substantial protection against some forms of leprosy. In Kenya, as in Uganda, the incidence of lepromatous leprosy in children is low. No conclusions can therefore be drawn as to the protective value of $B C G$ vaccination against more progressive forms of leprosy. For several reasons such an effect does not seem to be probable.

Leiker has frequently observed that lepromatous leprosy developed in individuals who had previously shown a specific positive tuberculin reaction or who suffered from tuberculosis long before the first signs of leprosy were seen. Although true epidemics of leprosy in New Guinea were found exclusively in areas with a low prevalence of tuberculosis, fairly high prevalences of leprosy were also found in areas with a high prevalence of tuberculosis. There was, however, a conspicuous difference in type distribution of leprosy between areas of low and of high prevalence of tuberculosis. In the former the prevalence of mild, high resistant tuberculoid leprosy, with only one or a few self-healing lesions, was markedly higher than in the latter 
areas. These findings suggest that tuberculosis may have reduced the incidence of high resistant tuberculoid leprosy, but that progressive forms of leprosy were not prevented.

Tuberculin-lepromin surveys have shown that part of the people with a specific positive tuberculin reaction do not show a strongly positive lepromin reaction and that in a small proportion of these individuals the lepromin reactions is even frankly negative. The size frequency distribution of lepromin reactions after ECG vaccination shows a similar pattern.

The lepromin reaction is regarded as a measure of the resistance to $M$. leprae at the moment of testing. The fact that in some individuals the lepromin reaction remains weak or is absent after tuberculosis infection or BCG vaccination, suggests that these individuals are not capable of developing a high resistance to $M$. leprae. When they become infected with $M$. leprae they will acquire an intermediate or a lepromatous form of leprosy. The majority of people are capable of reacting strongly to lepromin after BCG vaccination or tuberculosis infection and they will, after infection with $M$. leprae, not develop leprosy. Such individuals probably have a genetically determined potential resistance that has been transformed into an effective resistance with the aid of $M$. tuberculosis. If they had not been previously sensitised by infection with $M$. tuberculosis or by $\mathrm{BCG}$ vaccination they would, because of their potential resistance after leprosy infection, have developed effective resistance with the aid of $M$. leprae, and therefore would have shown a mild, self-healing, high resistant tuberculoid form of leprosy.

This hypothesis is further supported by the finding (Leiker, 1960) that the percentage of specific positive tuberculin reactions in patients who recently developed high resistant tuberculoid leprosy was lower than in healthy people of the same age and living in the same area. The evidence derived from tuberculin-lepromin studies corresponded with the epidemiological findings in New Guinea.

The size of the lepromin reaction in low resistant tuberculoid leprosy and in borderline tuberculoid leprosy, although on the average considerably smaller than in high resistant tuberculoid leprosy, is far from negligible. The size of the reaction is evidence of some resistance. This corresponds with the course of disease, a temporarily progressive course, not infrequently relapses, but ultimately followed by arrest of the disease. It is theoretically possible that in individuals with such a moderate degree of potential resistance a tubereulosis infection or BCG vaccination will produce just sufficient effective resistance that at least in some instances the few bacilli that have entered after incidental infection with $M$. leprae are destroyed rapidly before being able to produce symptoms of disease. Leiker has observed some patients with a specific positive tuberculin reaction who a few years later developed a single tuberculoid lesion. These lesions, however, showed typical signs of low resistant tuberculoid leprosy such as incomplete central healing, broad micro-papular edge and satellite lesions. This shows that early low reistant tuberculoid leprosy is not always prevented by previous tuberculosis infection.

It is regrettable that in the trial in Uganda no attempt was made to classify the patients with leprosy more accurately. It is often difficult to differentiate the early lesions in children on clinical symptoms alone, but a classification can frequently be based on lepromin reaction and histo-pathological examination. At present many of the children are receiving treatment and no conclusions can be drawn any more from the course of the disease. The incidence of lepromatous leprosy in children in Uganda is low. Unless the lepromin test is carried out in all patients so far discovered and lepromin test and histo-pathological examination are carried out in the new patients, the trial may not give an answer to the leading question whether BCG vaccination also protects against more progressive forms of leprosy or not.

\section{SUMMARY}

Re-surveys of schoolchildren in a highly endemic area in Kenya, 2 years after a BCG vaccination campaign, showed a marked decrease in the 
incidence of mild, non-lepromatous leprosy. No conclusion could be drawn as to the preventive effect of BCG vaccination against progressive forms of leprosy. Such an effect is not expected.

The BCG trial in Uganda too may not give an answer to the leading question of protection against progressive forms of leprosy because the incidence of such forms in children is low; accurate clinical classification is difficult in the early stage and many patients receive treatment in the early stage. It is suggested to carry out lepromin tests and histo-pathological examination in all patients for a more accurate classification in the uncharacteristic early stage.

\section{REFERENCES}

1. KinNeAr BRown, J. A., Stone, M. M. and SUtherLAND, I. BCG Vaccination of children against leprosy. First results of a trial in Uganda. $B r$. Med. .J. (1966), 1, 7-14.

2. LEIKER, D. L. Epidemiological and immunological surveys in Netherlands New Guinea. Lep. Rev. (1960), 31, 4, 241-259. 